\title{
Encuesta entomológica y caracterización de las viviendas con el vector de la enfermedad de Chagas, en aldea Chuchuapa, Santa Rosa, Guatemala
}

Entomological survey and characterization of houses with the vector of Chagas disease, in Chuchuapa village, Santa Rosa, Guatemala

\author{
David A. Girón ${ }^{1}$, Jimmy Saucedo ${ }^{1}$, Juan Ordoñez ${ }^{1}$, María Villagrán $^{1}$, Ángel Ojeda
} María Merlos ${ }^{1}$, Silvia Castro', Federico $\mathrm{Nave}^{2}$

${ }^{1}$ Facultad de Ciencias Médicas, ${ }^{2}$ Instituto de Investigaciones Químicas y Biológicas, Facultad de Ciencias Químicas y Farmacia, Universidad San Carlos de Guatemala, Guatemala

*Autor al que se dirige la correspondencia: dvd.gg93@gmail.com

Recibido: 13 de agosto 2019 / Revisión: 05 de febrero 2020 / Aceptado: 11 de junio 2020

\section{Resumen}

— n Guatemala en el 2015, el Ministerio de Salud Pública y Asistencia Social (MSPAS) reportó nuevos casos - de la enfermedad de Chagas en la región suroriental del país, siendo Santa Rosa uno de los departamentos endémicos en donde Triatoma dimidiata es el principal transmisor. Se estimó la infestación de T. dimidiata en la aldea Chuchuapa, municipio de Santa María Ixhuatán, de abril a junio de 2019, se calcularon los índices entomológicos y se caracterizaron intra y peridomiciliar 149 viviendas (79.67\% de las viviendas habitadas). Los datos se obtuvieron mediante un cuestionario mixto, en entrevista cara a cara y evaluación de las viviendas, por un equipo profesional experimentado, mediante el método y protocolo estándar hombre-hora. Se capturaron 20 triatominos en 10 viviendas, con un índice de infestación de 6.71\% (10/149), un índice de densidad de 13.42\% (20/149), 19 triatominos intradomiciliares y uno peridomiciliar, con un índice de infección natural por Trypanosoma cruzi intradomiciliar de 26.3\% (5/19), índice de infección natural por T. cruzi peridomiciliar de $100.0 \%$ (1/1) e índice de infección natural por T. cruzi en los triatominos capturados de $30.0 \%$ (6/20). Las viviendas infestadas presentaron techo de lámina, pared de bajareque con grietas y sin repello, piso de tierra y afiches en la pared, acumulación de leña u otros materiales tanto intra como peridomiciliar, convivencia con animales domésticos y tenencia de animales de corral. Los resultados representan un alto riesgo para la transmisión de la enfermedad de Chagas entre los habitantes de la aldea.

Palabras claves: Índices entomológicos, Factores de riesgo, Triatoma, Intradomiciliar, Peridomiciliar

\section{Abstract}

$I^{\prime}$ Guatemala in 2015, the Ministry of Public Health and Social Assistance reported new cases of Chagas disease in the southeastern region of the country, with Santa Rosa being one of the endemic departments where Triatoma dimidiata is the main transmitter. The infestation of T. dimidiata was estimated in the Chuchuapa village, municipality of Santa María Ixhuatán, from April to June 2019, the entomological indices were calculated and 149 dwellings were characterized intradomiciliary and peridomiciliary (79.67\% of the inhabited dwellings). The data were obtained through a mixed questionnaire, in a face-to-face interview and evaluation of the dwellings, by an experienced professional team, using the standard man-hour method and protocol. 20 triatomines were captured in 10 homes, with an infestation index of 6.71\% (10/149), a density index of $13.42 \%(20 / 149)$, 19 intradomiciliary triatomines and one peridomiciliary, with a natural infection index by intradomiciliary Trypanosoma cruzi of $26.3 \%(5 / 19)$, natural infection index by peridomiciliary T. cruzi of $100.0 \%(1 / 1)$ and natural infection index by T. cruzi in captured triatomines of $30.0 \%(6 / 20)$. The infested dwellings have a sheet roof, a wattle and daub wall with cracks and no wall plaster, dirt and posters on the wall, accumulation of firewood and other materials in both intradomiciliary and peridomiciliary, living with domestic animals and keeping poultry animals. The results represent a high risk for the transmission of Chagas disease among the villager. 


\section{Introduccion}

La enfermedad de Chagas es una enfermedad metaxénica, endémica y desatendida; según la Organización Mundial de la Salud (OMS), hay de seis a siete millones de personas afectadas a nivel mundial, siendo una enfermedad endémica en América Latina (OMS, 2018b). Se estima que alrededor de 10,000 personas fallecen al año a causa de sus manifestaciones clínicas y más de 25 millones se encuentran en riesgo de contraer la enfermedad en el mundo (Bustamante et al., 2015).

Respecto al vector de la enfermedad de Chagas en Latinoamérica se ha descrito una amplia gama de triatominos (26 de las 144 especies conocidas), donde Triatoma dimidiata y Rhodniux prolixus se trasforman en vectores recurrentes en las viviendas, especialmente en Costa Rica, Ecuador, Guatemala, El Salvador y Nicaragua (Quirós-Gómez et al., 2017). Triatoma dimidiata es el vector más importante en América Central, por lo que reducir la infestación domiciliaria es uno de los tres objetivos principales de la Iniciativa de los Países de Centro América (IPCA) (OMS, 2018a). Guatemala es un país endémico para esta enfermedad, por ello sigue siendo un tema de interés ya que existen estudios de distinta índole epidemiológica, entre ellos, uno realizado en el 2003 sobre la epidemiología de la enfermedad de Chagas donde se analizó la infestación por tres especies de vectores $T$. dimidiata, $R$. prolixus y Triatoma nitida, brindando información sobre la distribución vectorial en el territorio guatemalteco. $T$. dimidiata, vector con más probabilidad de poseer el parásito de Trypanosoma cruzi, se encontró diseminado en 16 de los 22 departamentos, incluyendo Santa Rosa (Monroy et al., 2003).

Los reportes documentados de esta enfermedad concuerdan que las características de las viviendas asociadas a la presencia del vector y la reinfestación de los vectores típicos de la región son los siguientes: paredes no repelladas o parcialmente repelladas con grietas, fabricadas de bajareque o adobe, piso de tierra, techo de palma, paja y lámina, con pocas habitaciones que favorezcan el hacinamiento, acumulación de material de construcción y presencia de animales domésticos, con localización en la periferia de gallineros o corrales (Bustamante et al., 2015; Quirós-Gómez et al., 2017). Así como materiales de construcción, granos, madera y/o leña para el consumo y cercanía con árboles frutales. También el uso de mosquiteros, pabellones, desorden dentro y fuera de las viviendas (Bustamante et al., 2015).
El Ministerio de Salud Pública y Asistencia Social (MSPAS), realizó un proyecto de control de vectores con la cooperación japonesa en el 2000 para reducir el riesgo de infección por medio de la eliminación de $R$. prolixus. Este consistió en la realización de campañas de fumigación con dos ciclos de rociamiento de insecticida residual y cubrió más del $98 \%$ de las casas en las aldeas identificadas (Hashimoto et al., 2012). A nivel internacional en el 2008 Guatemala fue reconocida y certificada por la eliminación de $R$. prolixus y el control de T. dimidiata. Sin embargo, se reportan casos continuamente de enfermedad, en el sur de Guatemala ya que $T$. dimidiata se mueve entre casas y aldeas con la frecuencia suficiente como para recolonizar, dificultando el control vectorial de la enfermedad. En el 2015 se estudió la estructura genética de la población de $T$. dimidiata recolectados de seis aldeas, dando a conocer que el uso de rociamientos simples sin otro método de intervención no es efectivo para la eliminación de este vector (Stevens et al., 2015).

Los últimos estudios realizados en la región de Santa Rosa, Guatemala, sobre la enfermedad de Chagas tienen una antigüedad de más de 20 años. Uno de los últimos descritos, se realizó en el 1997, donde se estudió una población de 1,085 habitantes residentes y originarios de Santa María Ixhuatán, que demostró una prevalencia de $126(11.61 \%)$ casos seropositivos para anticuerpos contra T. cruzi en la población estudiada (De León, 1997), además que el MSPAS en 2015 reportó casos en la región suroriental del país y según información del Departamento de Vectores, existe evidencia de la presencia del vector actualmente en la región estudia$\mathrm{da}$, ya que las condiciones del municipio son propicias para la proliferación del triatomino J. Zarceño, Jefe del departamento de vectores y enfermedades zoonóticas (comunicación personal, abril 2018). Durante los últimos tres años en el área de salud de Santa Rosa se reportaron 107 casos seropositivos de la enfermedad de Chagas, Epidemióloga del área de Santa Rosa (E. Reyes, comunicación personal, julio 2018).

El presente caso surgió de la necesidad de estudiar y describir la situación actual de la aldea Chuchuapa del municipio de Santa María Ixhuatán, departamento de Santa Rosa, en cuanto a la presencia del vector $T$. dimidiata, sus índices entomológicos y caracterización de las viviendas, por medio de la realización de una encuesta entomológica domiciliar, entrevista cara a cara al jefe o responsable de la vivienda mayor de 18 años y evaluación directa de las características de las viviendas, mediante el método estándar 
hombre-hora; con lo cual se espera contribuir a tener datos que alerten a las autoridades del MSPAS sobre el posible riesgo de adquirir la enfermedad de Chagas que puedan tener los habitantes de dicha comunidad.

\section{Presentación del caso}

Se realizó una encuesta entomológica de las viviendas habitadas de la aldea Chuchuapa, municipio de Santa María Ixhuatán, que se encuentra en la región suroriental del departamento de Santa Rosa, de la república de Guatemala, tomando como base el conteo poblacional del 2017 del Área de Salud de Santa Rosa. Los datos se obtuvieron del el jefe o responsables mayores de 18 años que estuvieran presentes en las viviendas al momento de la encuesta, la participación fue voluntaria, acompañada con firma de consentimiento informado o huella digital. Se recolectó la información mediante la realización de un cuestionario mixto conformado por una entrevista cara a cara y evaluación visual directa de las características intra y peridomiciliar en las viviendas.

Se buscó el vector en las áreas descritas de la vivienda mediante el método estándar hombre-hora. Fueron evaluadas 149 viviendas que permitieron su ingreso y correcta evaluación cumpliendo con los criterios establecidos, se evidenció una cobertura de las viviendas del $79.70 \%$ para la determinación de los índices entomológicos. Los triatominos encontrados fueron almacenados y transportados al Laboratorio de Entomología Aplicada y Parasitología (Lenap), posteriormente fueron evaluados macroscópicamente para determinar la especie y microscópicamente para identificar la infección por T. cruzi.

Se capturaron 20 triatominos que estaban distribuidos en 10 viviendas, de los cuales en seis de ellos se encontró el parásito $T$. cruzi durante la evaluación microscópica. La infestación del vector en las viviendas fue de $6.71 \%$ (10/149), con un índice de densidad de $13.42 \%$ (20/149), 19 triatominos intradomiciliares y uno peridomiciliar, el índice de infección natural por T. cruzi intradomiciliar fue de $26.3 \%$ (5/19), el cual se encuentra dentro de la media del país, y el índice de infección natural fue de $30.0 \%$ (6/20), el índice de infección natural del peridomicilio fue de $100 \%$, aunque solamente representa a un triatomino capturado el cual estaba infectado por T. cruzi.

De las viviendas que presentaron $T$. dimidiata, las características intradomicilio más comunes fueron: techo de lámina, pared de bajareque agrietadas o con fisuras, pisos de tierra, afiches en paredes, convivencia con animales domésticos durante el día y presencia de animales de corral, leña y otros materiales acumulados, ausencia de mosquitero y paredes sin repello. En tanto que las características peridomicilio más comunes fueron: animales de corral, animales domésticos, acumulación de leña y otros materiales. Tanto las características intradomiciliares como peridomiciliares fueron similares para las viviendas que no presentaron el vector (139/149), con la excepción que la mayoría de estas tenían pared de block, presencia de animales domésticos más que aves de corral intradomiciliarmente, así como reducida cantidad de viviendas con criaderos de animales peridomiciliarmente (Tablas 1 y 2).

\section{Discusión}

En este reporte, se encontró $T$. dimidiata como único vector de la enfermedad de Chagas, al igual que en estudios previos realizados en el departamento de Santa Rosa (Monroy et al., 2003); a nivel nacional se han reportado ocho especies de triatominos, pero con la erradicación de Rhodnius prolixus, T. dimidiata es sin duda el vector más importante (Peterson, Hashimoto et al., 2019). Naturalmente, esta especie ha sido encontrada en climas cálidos en bosques secos y húmedas, desde el nivel del mar hasta 2,000 m s. n. m. (Bustamante et al., 2009), la aldea Chuchuapa brinda un hábitat idóneo para la proliferación del vector, lo cual se demuestra por el índice de infestación de $6.71 \%$ encontrado, la cual es un porcentaje considerable y evidencia la presencia del vector como potencial transmisor de la enfermedad de Chagas. Esto representado en valores por cada 100 habitantes indica que prácticamente 7 de cada 100 viviendas se encuentran infestadas con el vector, con un índice de infección natural intradomicilio de $26.3 \%$, el cual podría ser considerado elevado, manifestando la falta de medidas de control en la construcción de viviendas y de la concientización de la población, pudiéndose considerar como una enfermedad desatendida en este departamento, ya que se han centrado los esfuerzos de control más que todo en Chiquimula y Jutiapa y se considera como de baja prioridad a nivel nacional (Peterson Hashimoto et al., 2019; Peterson, Yoshika, 2019); en Chiquimula, el índice de infestación observado en el 2000 fue de $21.13 \%$ habiendo disminuido en el 2007 a $2.0 \%$, luego de aplicar medidas de control, lo que evidencia lo indicado sobre la falta de atención al departamento de Santa Rosa, aunque este índice no llegó a ser tan elevado (Chávez, 2020). 
Tabla 1

Características intradomiciliares de las viviendas estudiadas

\begin{tabular}{|c|c|c|c|c|c|c|c|}
\hline \multicolumn{2}{|c|}{ Características en el intradomicilio } & \multicolumn{4}{|c|}{$\begin{array}{l}\text { Presencia de triatomino en la } \\
\text { vivienda }\end{array}$} & \multirow{3}{*}{$\begin{array}{c}\text { Total } \\
\mathrm{f}\end{array}$} & \multirow{3}{*}{$\begin{array}{c}\text { Total } \\
\%\end{array}$} \\
\hline \multirow{2}{*}{ Variable } & \multirow{2}{*}{ Categoría } & \multicolumn{2}{|c|}{ Sí } & \multicolumn{2}{|c|}{ No } & & \\
\hline & & $\mathrm{f}$ & $\%$ & $\mathrm{f}$ & $\%$ & & \\
\hline \multirow{3}{*}{ Techo } & Lámina & 9 & 6.04 & 130 & 87.25 & 139 & 93.29 \\
\hline & Cemento & 1 & 0.67 & 8 & 5.37 & 9 & 6.04 \\
\hline & Teja & - & - & 1 & 0.67 & 1 & 0.67 \\
\hline \multirow{5}{*}{ Pared } & Block & - & - & 67 & 44.97 & 67 & 44.97 \\
\hline & Bajareque & 9 & 6.04 & 23 & 15.44 & 32 & 21.48 \\
\hline & Tablas & - & - & 23 & 15.44 & 23 & 15.44 \\
\hline & Adobe & 1 & 0.67 & 4 & 2.68 & 5 & 3.36 \\
\hline & Otros & - & - & 22 & 14.77 & 22 & 14.77 \\
\hline \multirow{3}{*}{ Piso } & Tierra & 9 & 6.04 & 75 & 50.34 & 84 & 56.38 \\
\hline & Torta de cemento & 1 & 0.67 & 53 & 35.57 & 54 & 36.24 \\
\hline & Piso cerámico & - & - & 11 & 7.38 & 11 & 7.38 \\
\hline \multirow{2}{*}{ Afiches en paredes } & Sí & 9 & 6.04 & 113 & 75.84 & 122 & 81.88 \\
\hline & No & 1 & 0.67 & 26 & 17.45 & 27 & 18.12 \\
\hline \multirow{4}{*}{$\begin{array}{l}\text { Animales en el } \\
\text { intradomicilio }\end{array}$} & Animales domésticos & 5 & 3.36 & 64 & 42.95 & 69 & 46.31 \\
\hline & Animales de corral & 10 & 6.71 & 42 & 28.19 & 52 & 34.90 \\
\hline & Animales silvestres & - & - & 3 & 2.01 & 3 & 2.01 \\
\hline & Ninguno & - & - & 53 & 35.57 & 53 & 35.57 \\
\hline \multirow{8}{*}{$\begin{array}{l}\text { Materiales en el } \\
\text { intradomicilio }\end{array}$} & Leña & 7 & 4.70 & 59 & 39.60 & 66 & 44.30 \\
\hline & Otros & 5 & 3.36 & 60 & 40.27 & 65 & 43.62 \\
\hline & Granos & 3 & 2.01 & 38 & 25.50 & 41 & 27.52 \\
\hline & Madera & 1 & 0.67 & 9 & 6.04 & 10 & 6.71 \\
\hline & Lámina & - & - & 4 & 2.68 & 4 & 2.68 \\
\hline & Block & - & - & 3 & 2.01 & 3 & 2.01 \\
\hline & Teja & - & - & 1 & 0.67 & 1 & 0.67 \\
\hline & Ninguno & - & - & 3 & 2.01 & 3 & 2.01 \\
\hline \multirow{5}{*}{$\begin{array}{l}\text { Ausencia de medidas } \\
\text { protectoras }\end{array}$} & Mosquitero & 10 & 6.71 & 94 & 63.09 & 104 & 69.80 \\
\hline & Pabellón & 8 & 5.37 & 94 & 63.09 & 102 & 68.46 \\
\hline & Fumigación & 8 & 5.37 & 80 & 53.69 & 88 & 59.06 \\
\hline & Paredes de repello & 10 & 6.71 & 71 & 47.65 & 81 & 54.36 \\
\hline & Ninguno & - & - & 3 & 2.01 & 3 & 2.01 \\
\hline \multirow{3}{*}{$\begin{array}{l}\text { Convivencia con } \\
\text { animales domésticos }\end{array}$} & Día & 10 & 6.71 & 114 & 76.51 & 124 & 83.22 \\
\hline & Noche & - & - & 18 & 12.08 & 18 & 12.08 \\
\hline & No aplica & - & - & 15 & 10.07 & 15 & 10.07 \\
\hline
\end{tabular}


Tabla 2

Características peridomiciliares de las viviendas estudiadas

\begin{tabular}{|c|c|c|c|c|c|c|c|}
\hline \multicolumn{2}{|c|}{ Características en el peridomicilio } & \multicolumn{4}{|c|}{ Presencia de triatomino en la vivienda } & \multirow{3}{*}{$\begin{array}{c}\text { Total } \\
\mathrm{f}\end{array}$} & \multirow{3}{*}{$\begin{array}{c}\text { Total } \\
\%\end{array}$} \\
\hline \multirow{2}{*}{ Variable } & \multirow{2}{*}{ Categoría } & \multicolumn{2}{|c|}{ Sí } & \multicolumn{2}{|c|}{ No } & & \\
\hline & & $\mathrm{f}$ & $\%$ & $\mathrm{f}$ & $\%$ & & \\
\hline \multirow{4}{*}{$\begin{array}{l}\text { Animales en el } \\
\text { peridomicilio }\end{array}$} & Animales domésticos & 7 & 4.70 & 76 & 51.01 & 83 & 55.70 \\
\hline & Animales de corral & 8 & 5.37 & 75 & 50.34 & 83 & 55.70 \\
\hline & Animales silvestres & - & - & 3 & 2.01 & 3 & 2.01 \\
\hline & Ninguno & - & - & 25 & 16.78 & 25 & 16.78 \\
\hline \multirow{5}{*}{$\begin{array}{l}\text { Criaderos de } \\
\text { animales }\end{array}$} & Gallineros & 4 & 2.68 & 37 & 24.83 & 41 & 27.52 \\
\hline & Corrales & 2 & 1.34 & 20 & 13.42 & 22 & 14.77 \\
\hline & Jaulas & - & - & 8 & 5.37 & 8 & 5.37 \\
\hline & Cochiqueras & 2 & 1.34 & 6 & 4.03 & 8 & 5.37 \\
\hline & Ninguno & 4 & 2.68 & 76 & 51.01 & 80 & 53.69 \\
\hline \multirow{8}{*}{$\begin{array}{l}\text { Materiales en el } \\
\text { peridomicilio }\end{array}$} & Leña & 9 & 6.04 & 111 & 74.50 & 120 & 80.54 \\
\hline & Granos & 1 & 0.67 & 14 & 9.40 & 15 & 10.07 \\
\hline & Madera & 1 & 0.67 & 8 & 5.37 & 9 & 6.04 \\
\hline & Lámina & - & - & 4 & 2.68 & 4 & 2.68 \\
\hline & Block & 2 & 1.34 & 2 & 1.34 & 4 & 2.68 \\
\hline & Ninguno & - & - & 3 & 2.01 & 3 & 2.01 \\
\hline & Teja & - & - & 2 & 1.34 & 2 & 1.34 \\
\hline & Otros & 5 & 3.36 & 35 & 23.49 & 40 & 26.85 \\
\hline
\end{tabular}

De los 20 vectores recolectados, 19 (95\%) fueron encontrados intradomiciliarmente, lo que concuerda con un estudio realizado en Colombia donde el 93\% de los triatominos fueron capturados en ambientes intradomiciliarios (Cortés \& Suárez, 2005). El índice de infestación fue de $6.71 \%$ indicando el índice de infestación del vector en esta región, el índice de densidad muestra que en 13 de cada 100 viviendas se encontrará el triatomino, de los cuales $30 \%$ estará infectado con T. cruzi según el índice de infección natural, dato superior al reportado por Juarez y colaboradores (2018) para el departamento de Jutiapa en 2011 que fue de $26.9 \%$ de infestación natural, aunque con una prevalencia de $20.5 \%$ y también superior al índice de infestación de $24.0 \%$ en El Carrizal, Jutiapa (Cahan et al., 2019). Por otra parte, según los índices reportados por Lima-Cordón y colaboradores (2018), para el municipio de Olopa, departamento de Chiquimula para el 2011 se reportó un porcentaje de infección natural muy infe- rior $(8 \%)$ al encontrado en este reporte. Aunque para el 2017, se reportaba un porcentaje de infestación de $2.75 \%$ en comunidades de ambos departamentos, luego de un programa de vigilancia del vector y aplicación de insecticida a domicilios (Peterson, Hashimoto et al., 2019), lo cual no se ha realizado en la aldea reportada en este artículo.

Los índices de densidad y de infección natural, se explican en parte, por las condiciones de las viviendas, tal y como indican Bustamante y colaboradores (2009) en una investigación sobre factores de riesgo para infestación intradomiciliaria del vector de la enfermedad de Chagas que concluye que, si las viviendas son antiguas, desordenadas y con condiciones precarias puede incrementar la probabilidad que tengan exuvias y rastros de deyecciones que las viviendas nuevas o limpias. En este reporte se observa que, en general, las condiciones intradomiciliares son similares entre las viviendas con la presencia del vector y las que no hubo 
capturas, aunque se hayan evidenciado deyecciones del vector en paredes y hasta algunas exuvias; por lo cual se puede concluir que las condiciones de las viviendas de la aldea Chuchuapa son favorables para la presencia del vector a pesar de no capturarlo.

Con respecto a las características intradomiciliares de las viviendas, estas presentaron condiciones que en estudios tanto en Guatemala como en Colombia, se han asociado a la presencia o al incremento de la prevalencia del vector, como techo de lámina, paredes de bajareque, piso de tierra, ausencia de medidas protectoras y afiches, presencia de animales de corral y domésticos, la convivencia con ellos durante el día y materiales acumulados en el intradomicilio de manera desordenada o desorganizada (Bustamante et al., 2015; Quirós-Gómez et al., 2017; Salvatella, 2016). Pero especial atención debe ponerse en los casos de viviendas con paredes no repelladas, hechas de bajareque con afiches y desorganizadas, lo que se ha reportado que produce un incremento probable de la presencia de T. dimidiata (Bustamante et al., 2014; Bustamante et al., 2009; 2015; Quirós-Gómez et al., 2017; Salvatella, 2016; Sandoval-Ruiz et al., 2014). Las condiciones de las viviendas de la aldea Chuchuapa concuerdan con las encontradas en el Chaco paraguayo caracterizadas por la falta de limitación entre el área silvestre y las viviendas, resaltando la acumulación de leña (Sánchez et al., 2016) como el material predominante.

Las características peridomiciliares de las viviendas de la aldea estudiada concuerdan con las descritas por Hashimoto y colaboradores (2012) en Guatemala, quienes mencionan que a nivel peridomicilio los corrales de aves, gallineros y criaderos de animales domésticos localizados aproximadamente de 2 a 30 $m$ de la vivienda fueron determinantes para encontrar el vector; reportes similares se han tenido en varios estudios con relación a este tipo de condiciones peridomicilares (Bustamante et al., 2009, 2015; De León, 1997, Gurgel-Gonçalves et al., 2010; De Urioste-Stone et al., 2015; Quirós-Gómez et al., 2017) y que han sido asociadas al incremento probable de la presencia del vector (Calvillo et al., 2014; Dumonteil et al., 2013).

En un estudio reciente, se reportaron para dos aldeas del departamento de Jutiapa índices de infestación elevados (entre 25 y $35 \%$ ), asociados a factores intradomiciliares similares a los encontrados en este estudio (paredes de adobe y bajareque, animales intradomicilares y piso de tierra), por lo que el índice de infestación de $6.71 \%$ encontrado en este reporte, si bien es importante, se mantiene entre el rango esperado como de baja infestación, pero las condiciones de las viviendas son un riesgo aún latente que podría contribuir al aumento del índice de infestación (Penados et al., 2020). Investigaciones realizadas en el municipio de Comapa, departamento de Jutiapa, han demostrado la reducción de la infestación domiciliar por triatominos aplicando estrategias de control que conllevan mejoras a las viviendas y una activa participación comunitaria (Castro-Arroyave et al., 2020).

El índice de infestación de T. dimidiata en las viviendas, así como el índice de infección por T. cruzi que presentan los triatominos, las paredes de las viviendas agrietadas y fisuradas, la falta de métodos para contrarrestar la propagación del vector como repellar las paredes y mejorar la infraestructura de las viviendas, así como las condiciones de las viviendas tanto intra como peridomiciliarmente, el acúmulo de materiales dentro y fuera de las viviendas, representan un alto riesgo para la transmisión de la enfermedad de Chagas entre los habitantes de la aldea Chuchuapa, municipio de Santa María Ixhuatán.

\section{Referencias}

Bustamante, D. M., De Urioste-Stone, S. M., Juárez, J.G., \& Pennington, P. M. (2014). Ecological, social and biological risk factors for continued Trypanosoma cruzi transmission by Triatoma dimidiata in Guatemala. PLoS ONE, 9(8), Article e104599. https://doi.org/10.1371/journal. pone.0104599

Bustamante, D. M., Hernández, M. M., Torres, N., Zúñiga, C., Sosa, W., Abrego V, \& Monroy, M. C. (2015). Information to act: Household characteristics are predictors of domestic infestation with the Chagas vector Triatoma dimidiata in Central America. American Journal of Tropical Medicine and Hygiene, 93(1), 97-107. https://doi.org/10.4269/ajtmh.14-0596

Bustamante, D. M., Monroy, C., Pineda, S., Rodas, A., Castro, X., Ayala, V., Quiñonez, J., Moguel, B., \& Trampe, R. (2009). Risk factors for intradomiciliary infestation by the Chagas disease vector Triatoma dimidiata in Jutiapa, Guatemala. Cadernos de Saúde Pública, 25(Suppl 1), S83-S92. https://doi.org/10.1590/ S0102-311X2009001300008

Cahan, S. H., Orantes, L. C., Wallin, K. F., Hanley, J. P., Rizzo, D. M., Stevens, L., Dorn, P. L., Rodas, 
A., \& Monroy, C. (2019). Residual survival and local dispersal drive reinfestation by Triatoma dimidiata following insecticide application in Guatemala. Infection, Generics and Evolution, 74. 104000. https://doi.org/10.1016/j. meegid.2019.104000

Calvillo, M. J., López, M. R., \& Rivera, M. E. (2014). Prevalencia de la enfermedad de Chagas en niños de 7 a 14 años en el municipio de Olopa, departamento de Chiquimula, Guatemala [Tesis de licenciatura, Universidad de San Carlos de Guatemala]. http://www.repositorio.usac.edu. gt/2120/1/06_3685.pdf

Castro-Arroyave, D., Monroy, M. C., \& Irurita, M. I. (2020). Integrated vector control of Chagas disease in Guatemala: A case of social innovation in health. Infectious Diseases of Poverty, 9, 25. https://doi.org/10.1186/s40249-020-00639-w,

Chávez, E. (2020). Evolución de la enfermedad de Chagas e infestación con triatominos en Chiquimula, Guatemala, 2000-2018. Revista Médica (Colegio de Médicos y Cirujanos de Guatemala), 159(2), 85-90. https://doi. org/10.36109/rmg.v159i2.254

Cortés, L. A., \& Suárez, H. A. (2005). Triatominos (Reduviidae: Triatominae) en un foco de enfermedad de Chagas en Talaigua Nuevo (Bolívar, Colombia). Biomedica, 25(4), 568-574. https://doi.org/10.7705/biomedica.v25i4.1383

De León, M. P. (1997). Estudio clínico, serológico y epidemiológico de la enfermedad de Chagas en Santa María Ixhuatán, Santa Rosa [Tesis de licenciatura, Universidad de San Carlos de Guatemala]. http://biblioteca.usac.edu.gt/ tesis/06/06_1845.pdf

De Urioste-Stone, S. M., Pennington, P. M., Pellecer, E., Aguilar, T. M., Samayoa, G., Perdomo, H. D., Enríquez, H., \& Juárez, J. G. (2015). Development of a community-based intervention for the control of Chagas disease based on peridomestic animal management: an eco-bio-social perspective. Transactions of the Royal Society of Tropical Medicine and Hygiene, 109(2), 159-167. https:// doi.org/10.1093/trstmh/tru202

Dumonteil, E., Nouvellet, P., Rosecrans, K., RamírezSierra, M. J., Gamboa-León, R., Cruz.Chang, V., Rosado-Vallado, M., \& Gourbière, S. (2013).
Eco-bio-social determinants for house infestation by non-domiciliated Triatoma dimidiata in the Yucatan Peninsula, Mexico. PLoS Neglected Tropical Diseases, 7(9), Article e2466. https://doi.org/10.1371/journal.pntd.0002466

Gurgel-Gonçalves, R., Alves, F., Pereira, I., \& Cavalcante, R. R. (2010). Distribución geográfica, infestación domiciliaria e infección natural de triatóminos (Hemíptera: Reduviidae) en el Estado de Piauí, Brasil, 2008. Revista Pan-Amazônica de Saúde, 1(4), 57-64. https://doi.org/10.5123/S217662232010000400009

Hashimoto, K., Álvarez, H., Nakagawa, J., Juarez, J., Monroy, C., Cordón C., \& Gil, E. (2012). Vector control intervention towards interruption of transmission of Chagas disease by Rhodnius prolixus, main vector in Guatemala. Memórias do Instituto Oswaldo Cruz, 107(7), 877-887. https://doi.org/10.1590/S0074-02762012000700007

Juarez, J. G., Pennington, P. M., Bryan, J. P., Klein, R. E., Beard, C. B., Berganza, E., Rizzo, N., \& Cordon-Rosales, C. (2018). A decade of vector control activities: Progress and limitations of Chagas disease prevention in a region of Guatemala with persistent Triatoma dimidiata infestation. PLoS Neglected Tropical Diseases, 12(11), Article e0006896. https://doi. org/10.1371/journal.pntd.0006896

Lima-Cordón, R. A., Stevens, L., Solórzano Ortíz, E., Rodas, G. A., Castellanos, S., Rodas, A., Concepción, V., \& Monroy, M. A. (2018). Implementation science: Epidemiology and feeding profiles of the Chagas vector Triatoma dimidiata prior to Ecohealth intervention for three locations in Central America. PLoS Neglected Tropical Diseases, 12(11), Article e0006952. https://doi.org/10.1371/journal.pntd.0006952

Ministerio de Salud Pública y Asistencia Social. (2015). Informe anual de situación de enfermedades transmisibles y no transmisibles prioritarias de vigilancia epidemiológica. 52-55. http://epidemiologia.mspas.gob.gt/ files/publicaciones\%202017/Desarollo/., PRIORIDADES DE VIGILANCIA EPI 1de1.pdf

Monroy, C., Rodas, A., Mejía, M., Rosales R., \& Tabaru Y. (2003). Epidemiology of Chagas disease in Guatemala: Infection rate of Triatoma 
dimidiata, Triatoma nitida and Rhodnius prolixus (Hemiptera, Reduviidae) with Trypanosoma cruzi and Trypanosoma rangeli (Kinetoplastida, Trypanosomatidae). Memórias do Instituto Oswaldo Cruz, 98(3), 305-310. https://doi. org/10.1590/S0074-02762003000300003

Organización Mundial de la Salud. (2018a). Chagas disease (american trypanosomiasis), epidemiology. http://www.who.int/chagas/ epidemiology/en/

Organización Mundial de la Salud. (2018b). La enfermedad de Chagas (tripanosomiasis americana). http://www.who.int/es/news-room/ fact-sheets/detail/chagas-disease-(americantrypanosomiasis)

Quirós-Gómez, O., Jaramillo, N., Angulo, V., \& Parra-Henao, G. (2017). Triatoma dimidiata en Colombia; distribución, ecología e importancia epidemiológica. Biomédica, 37(2), 274-285. https://doi.org/10.7705/biomedica.v37i2.2893

Penados, D., Pineda, J., Catalan, M., Avila, M., Stevens, L., Agreda, E., \& Monroy, C. (2020). Infestation dynamics of Triatoma dimidiata in highly deforested tropical dry forest regions of Guatemala. Memórias do Instituto Oswaldo Cruz, 115, Article e200203. https://doi. org/10.1590/0074-02760200203

Peterson, J. K., Hashimoto, K., Yoshioka, K., Dorn, P. L., Gottdenker, N. L., Caranci, A., Stevens, L., Saldaña, A., Rodriguez, S., \& Monroy, C. (2019). Chagas disease in Central America: Recent findings and current challenges in vector ecology and control. Current Tropical Medicine Reports, 6(2), 76-91. https://doi.org/10.1007/s40475-019$00175-0$
Peterson, J. K., Yoshika, K., Hashimoto, K., Caranci, A., Gottdenker, N., Montroy, C., Saldaña, A., Rodriguez, S., Dorn, \& P., Zúñiga, C. (2019). Chagas disease epidemiology in Central America: An update. Current Tropical Medicine Reports, 6(2), 92-105. https://doi.org/10.1007/ s40475-019-00176-z

Salvatella, R. (2016). Chagas en Uruguay, 1937-2016 Información básica para su prevención, control y atención. Archivos de Pediatría del Uruguay, $87(1), 49-52$

Sánchez, Z., Russomando, G., Chena, L., Nara, E., Cardozo, E., Paredes, B., \& Ferreira, E. (2016) Triatoma sordida: indicadores de adaptación y transmisión de Trypanosoma cruzi en intradomicilio del Chanco Paraguayo. Memorias del Instituto de Investigaciones en Ciencias de la Salud, 14(3), 96-101. https://doi.org/10.18004/ Mem.iics/1812-9528/2016.014(03)96-101

Sandoval-Ruiz, C. A., Guevara, R., \& Ibáñez-Bernal, S. (2014). Household risk factors associated to infestation of Triatoma dimidiata, the Chagas disease vector in Central Region of Veracruz, Mexico. Salud Pública de México, 56(2), 213-220

Stevens, L., Monroy, M. C., Rodas, A. G., Hicks, R. M., Lucero, D. E., Lyons L. A., \& Dorn, P. L. (2015). Migration and gene flow among domestic populations of the Chagas insect vector Triatoma dimidiata (Hemiptera: Reduviidae) detected by microsatellite loci. Journal of Medical Entomology, 52(3), 419-428. https://doi. org/10.1093/jme /tjv002 